\title{
Riqueza, composición y abundancia de aves del Campus Universitario de la Universidad Nacional de Asunción, San Lorenzo, Paraguay*
}

\author{
F. Ortiz ${ }^{1}$ K. Núñez ${ }^{1}$ y L. Amarilla ${ }^{2}$ \\ ${ }^{1}$ Departamento de Biología de la Facultad de Ciencias Exactas y Naturales. FACEN. E-mail: fno-py@hotmail.com; ranitapy@hotmail.com \\ ${ }^{2}$ Museo Nacional de Historia Natural del Paraguay MNHNP - Secretaria de Ambiente SEAM. E-mail: seferuziel@gmail.com
}

RESUMEN. Entre septiembre de 2014 y marzo de 2015 se realizó un estudio de avistamiento de aves en el Campus Universitario de la Universidad Nacional de Asunción, San Lorenzo, Paraguay. El objetivo fue analizar su diversidad en las estaciones de primavera y verano, a través de parámetros como riqueza, abundancia y composición de aves en el campus. La riqueza fue de 77 especies, que corresponden a 27 familias distribuidas en 13 órdenes. En cuanto a la abundancia resultaron abundantes 24 especies, diez especies comunes, siete especies poco comunes, 18 especies escasas y 15 especies raras. Dada esta gran diversidad de aves dentro del campus, éste puede ser considerado un refugio ecológico para las aves y un interesante punto de observación dentro del área urbana.

Palabras clave: Avifauna, ecosistemas modificados, habitas urbanos.

\begin{abstract}
Richness, community composition and abundance of birds of Campus Universitario, Universidad Nacional de Asunción, San Lorenzo, Paraguay. Between September 2014 and March 2015, we conducted a bird watching study in the Campus Universitario of the National University of Asuncion, San Lorenzo, Paraguay. Our objective was to analyze spring and summer diversity through parameters like species richness, abundance and composition of birds on the study area. Species richness was 77 species, corresponding to 27 families in 13 orders. Twenty-four species were abundant, ten common, seven uncommon, 18 scarce and 15 rare. Given this great diversity of birds within the study area, this can be considered as an ecological refuge for birds, and an interesting observation point within the urban area.
\end{abstract}

Key words: Birds, modified ecosystems, urban habitats.

*Trabajo presentado en el marco de la XVI Reunión Argentina de Ornitología (La Plata, 9 al 12 de septiembre de 2015) organizada por la Sección Ornitología, División Zoología Vertebrados, Museo de La Plata, Facultad de Ciencias Naturales y Museo, Universidad Nacional de La Plata. 


\section{Introducción}

Aunque muchas especies son afectadas negativamente cuando se modifican los hábitats naturales, algunas pueden adaptarse a los cambios realizados por el hombre, o incluso pueden aparecer nuevas especies, debido a la oferta diferencial de recursos (Maragliano et al. 2009). En este sentido, los estudios ecológicos en áreas urbanas son necesarios, ya que las ciudades continúan creciendo en superficie y número de habitantes, por lo que la restauración y conservación de la biodiversidad en ambientes urbanos se vuelve muy importante (Savard et al. 2000).

Las aves constituyen un grupo estudiado con frecuencia en los centros urbanos (Takano \& Castro 2007, Juri \& Chani 2009, Londoño-Betancourth 2011), debido a que el muestreo es más sencillo en relación a otros grupos de vertebrados y se pueden medir parámetros comunitarios como riqueza, composición y abundancia, que permiten establecer comparaciones con hábitats menos impactados (Savard et al. 2000, González et al. 2007).

Los estudios de avifauna en las ciudades coinciden en señalar que la riqueza de especies de aves disminuye conforme se incrementa la urbanización (Chace \& Walsh 2006, Faggi \& Perepelizin 2006). Las especies que logran adaptarse, son aquellas que pueden utilizar los recursos disponibles para su alimentación, reproducción, anidación y descanso, como por ejemplo Columba livia y Passer domesticus (Leveau \& Leveau 2004).

Dentro de las ciudades, los campus universitarios son sitios interesantes para realizar estudios sobre las comunidades de aves, porque generalmente conservan parches con más vegetación que la matriz circundante (Stiles 1990), combinándose especies de plantas nativas y exóticas. En Paraguay, del Campus Universitario de la Universidad Nacional de Asunción (UNA) se conocen los trabajos de Escobar \& De Salomón (1983), quienes registraron 41 especies de aves, Rodríguez (1986) citó a 42 especies y un estudio puntual más reciente presentó una lista de aves compuesta por 24 especies (Álvarez et al. 2012).

Para enriquecer el conocimiento sobre la avifauna del campus universitario de la UNA, se realizó este trabajo, cuyos objetivos fueron determinar la riqueza, la composición y la abundancia de aves diurnas del Campus Universitario de Asunción, durante las estaciones de primavera y verano.

\section{Materiales y métodos}

\section{Área de estudio}

El estudio se llevó a cabo entre los meses de septiembre de 2014 y marzo de 2015, abarcando las estaciones de primavera y verano, dentro del Campus Universitario (UNA), ubicada en el distrito de San Lorenzo, Paraguay ( $\left.26^{\circ} 20^{\prime} 08^{\prime \prime} \mathrm{S} ; 57^{\circ} 31^{\prime} 14^{\prime \prime} \mathrm{O}\right)$. Este predio es contiguo al casco urbano de la ciudad del mismo nombre, distante a $11 \mathrm{~km}$ del centro de la ciudad de Asunción, con una superficie de 187,6 ha (Ramírez 2005). La vegetación del campus se distribuye en parches muy pequeños, sin continuidad, con predominancia de árboles de las especies nativas Tabebuia sp, Jacaranda sp, Cordia sp, Albizia sp y exóticas como Eucalyptus sp. Además existen pastizales, canchas, campos de experimentación agrícola, edificaciones y caminos de acceso (De Jesús 2006).

En el área de estudio se establecieron en total 10 puntos de conteo para llevar a cabo las observaciones de las especies de aves que habitan en el predio del campus universitario (Figura 1).

\section{Toma de datos}

En los meses de septiembre y octubre 2014 se realizó un muestreo inicial, durante un total de 15 días consecutivos, estableciendo tres puntos de conteo. El recorrido se realizó por las mañanas de 6:30 a 9:00 horas, aproximadamente. Se utilizó como base la metodología de conteo por punto de radio fijo 
propuesta por Ralph et al. (1996), que consiste en la permanencia de 5-10 minutos en cada punto de conteo, registrando a todas las aves vistas y/o escuchadas, ampliando la permanencia en los puntos de conteo en algunas pocas ocasiones.

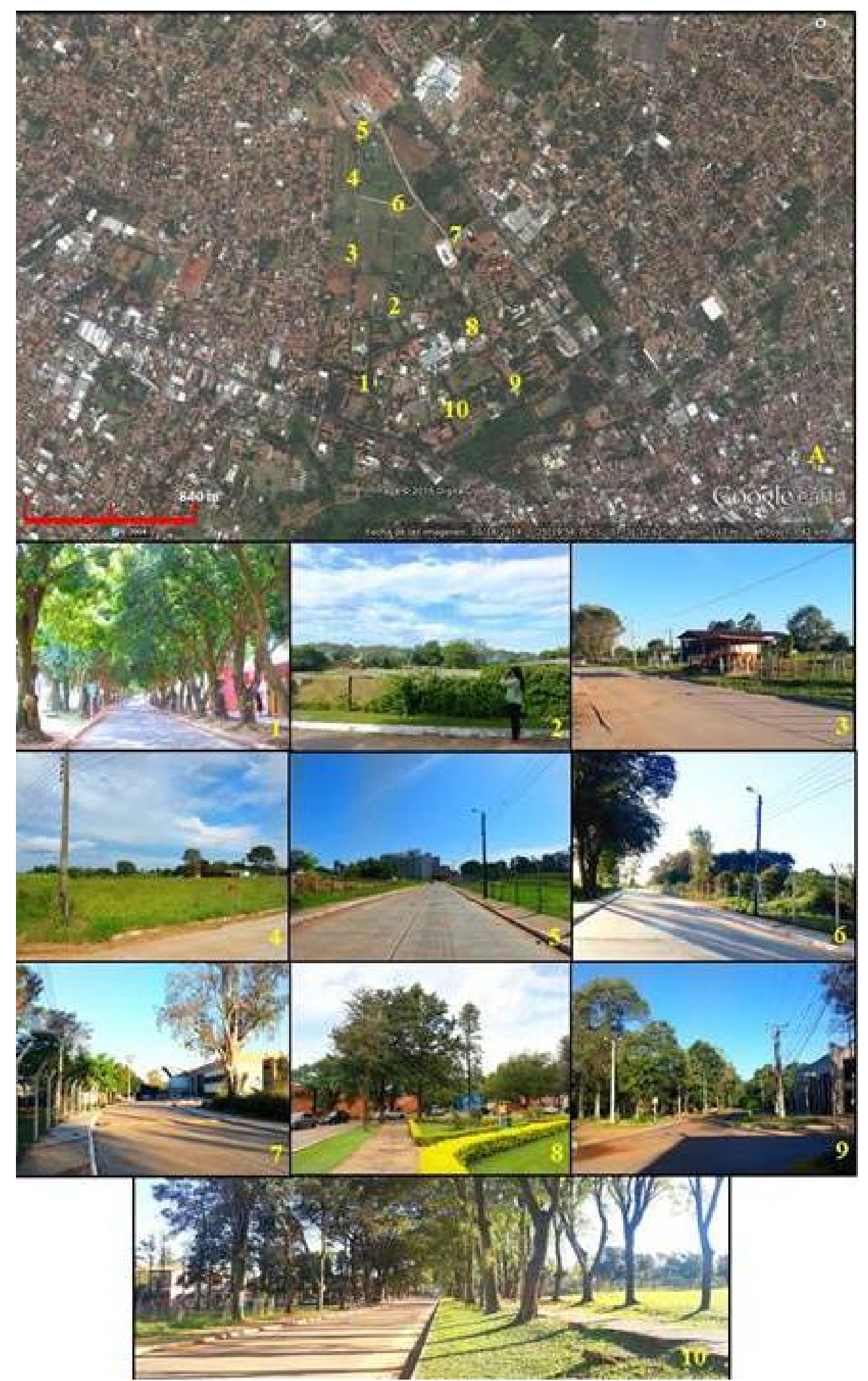

Figura 1. Localización del área de estudio. A) Mapa del campus universitario con los puntos de conteo, 1) zona de veterinaria, 2) zona de piscicultura, 3) zona de establos de veterinaria, 4) zona de pastizal, 5) zona de hospital, 6) zona de agronomía experimental, 7) zona de Facultad de Ingeniería - IICS, 8) zona de rectorado, 9) zona de facultad de Ciencias Agrarias, 10) zona de Facultad de Ciencias Económicas. 
A partir de este muestreo inicial, se ampliaron a diez los puntos de conteo, para una mejor representatividad de la diversidad de aves del campus. La toma de datos continuó desde noviembre hasta marzo, totalizando 32 días de esfuerzo de muestreo (un día de esfuerzo implica 10 puntos de conteo en el campus). Entre los puntos de conteo existió una distancia mínima de 200 metros.

Los registros se realizaron dos veces por semana, desde las 6:30 hasta las 11:00 horas aproximadamente. En algunas pocas ocasiones se realizaron registros fuera del horario y periodo de estudio establecido.

Las observaciones se llevaron a cabo utilizando binoculares 10 x 50 de la marca Bushnell y una cámara digital marca LUMIX 24X para el registro fotográfico de las aves. Para la identificación de las especies se utilizó las guías de aves de Narosky \& Yzurieta (2006), Guyra Paraguay (2002) y De la Peña \& Rumboll (1998). La nomenclatura taxonómica fue actualizada según la base de datos electrónica Avibase (2015) y Clements (2015).

\section{Análisis de datos}

Se realizaron tablas con la identificación de todas las especies, las familias y los órdenes a las que pertenecen. Con los datos del muestreo sistemático, se elaboró una curva de acumulación de especies para evaluar la riqueza según el esfuerzo de muestreo. Se utilizaron los estimadores no paramétricos Chao 2, Jack 1 y Jack 2 (Moreno 2001). Estos análisis se realizaron con el software EstimateS (Colwell 2013).

El análisis de la abundancia se basó en la frecuencia de detección de las especies en el área (Marín 2005). También se utilizó un índice de importancia relativa (IIR), modificado a partir de Gatto et al. (2005), donde la abundancia fue definida por la cantidad de sitios que ocupa una especie y la cantidad de días en los que estuvo presente en el muestreo. Con este índice se clasificó a las especies como abundantes (IIR $=76-100$ ), comunes (IIR $=51-75$ ), poco comunes (IIR $=26-50$ ), escasas (IIR $=10-25$ ) y raras (IIR <10) (Marín 2005). Además se clasificó a las especies de acuerdo a las estaciones en que fueron detectadas. Para determinar la categoría de amenaza de cada especie se siguió a IUCN (2015) y las Resoluciones de la Secretaría del Ambiente (SEAM) No 2242/06 y 2243/06.

\section{Resultados}

Se registraron un total de 77 especies de aves, que corresponden a 27 familias distribuidas en 13 órdenes (Tabla 1). El orden con más representatividad de especies fue Passeriformes, compuesto por 43 especies. Las familias representadas por más especies fueron Thraupidae con 12 especies y Tyrannidae con 9 especies. De las 77 especies, dos especies fueron exóticas (Passer domesticus y Columba livia) y tres fueron únicamente registradas en el muestreo ocasional (Falco sparverius, Milvago chimachima y Phacellodomus ruber) y no formaron parte de los análisis de riqueza y abundancia. Ninguna especie revistió categoría de amenaza.

La curva acumulada de riqueza de especies indicó que después de 32 días de muestreo, la curva llegó a una asíntota (Figura 2). La riqueza observada (74 especies) y las estimadas por los índices Chao 2 (76 especies), Jack 1 ( 81 especies) y Jack 2 (76 especies) fueron muy similares, demostrando la eficiencia del muestreo sistemático. Estos estimadores indicaron que se conoce hasta el $97 \%$ de la riqueza real del lugar (Chao 2), el 91\% Jack 1 y el 97\% con el estimador de Jack 2 (Figura 2).

Según el índice de importancia relativa (IIR) resultaron 24 especies abundantes, 10 especies comunes, 7 especies poco comunes, 18 especies escasas y 15 especies raras (Tabla 1). Sesenta especies (78\%) fueron registradas durante todo el periodo de estudio, 8 especies (10\%) aparecieron sólo durante la primavera y 9 especies sólo durante el verano (12\%) (Tabla 1). Probablemente las especies que sólo se observaron en una estación son aquellas que esporádicamente han visitado el 
campus universitario aprovechando el predio del campus en forma ocasional para la búsqueda de alimento y/o descanso.

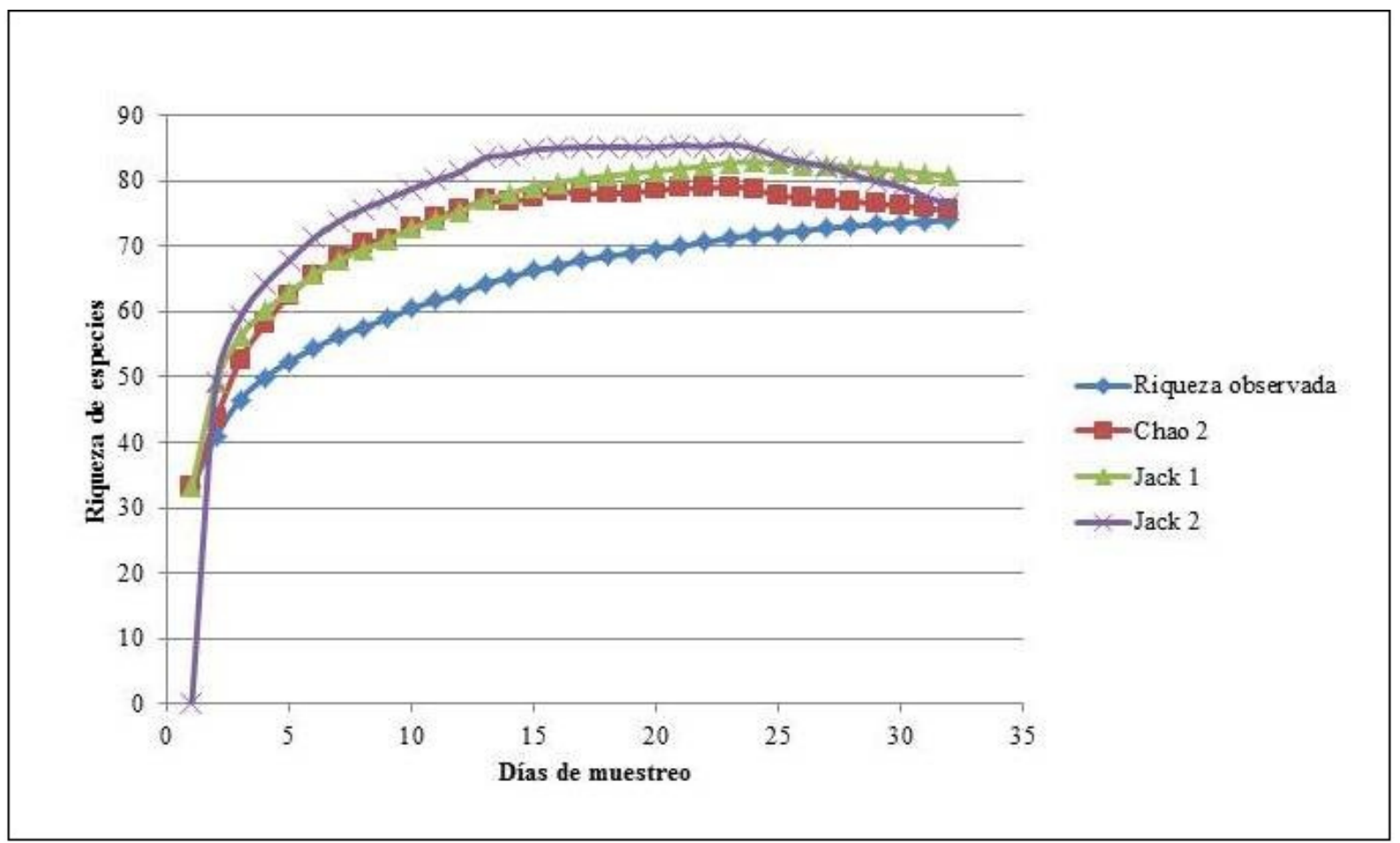

Figura 2. Curva de acumulación de especies de aves del campus universitario de la UNA basada en la riqueza observada y estimadores Chao2, Jack 1 y Jack 2 (Moreno 2001) en función a los días de muestreo.

Tabla 1: Especies de aves del Campus Universitario (Universidad Nacional de Asunción). Se indica el Orden, Familia y Especie, nombre en guaraní y en español, estación en la cual la especie fue registrada y el índice de importancia relativa.

\begin{tabular}{|c|c|c|c|c|c|}
\hline $\begin{array}{l}\text { Orden y } \\
\text { Familia }\end{array}$ & Especie & Nombre guaraní & Nombre español & Estación & IIR \\
\hline \multicolumn{6}{|c|}{ Accipitriformes } \\
\hline \multirow{3}{*}{ Accipitridae } & Gampsonyx swainsonii ${ }^{(z)}$ & Taguato'i & Milano chico & $\mathrm{V}$ & 5 \\
\hline & Rupornis magnirostris ${ }^{(a, b)}$ & Yndaje & Taguato común & PV & 55 \\
\hline & Parabuteo unicinctus $^{(z)}$ & Yryvutinga & Águila negra & PV & 5 \\
\hline \multirow{2}{*}{ Cathartidae } & Cathartes aura ${ }^{(z)}$ & Yryvu akã virai & Cuervo cabeza roja & PV & 10 \\
\hline & Coragyps atratus ${ }^{(a, b)}$ & Yryvu hũ & Cuervo negro & PV & 14 \\
\hline \multicolumn{6}{|l|}{ Apodiformes } \\
\hline \multirow{3}{*}{ Trochilidae } & Chlorostilbon lucidus ${ }^{(a, b)}$ & Mainumby hovyũ & Picaflor verde & PV & 81 \\
\hline & Heliomaster furcifer ${ }^{(z)}$ & Minumby jetapa & Picaflor de barbijo & PV & 74 \\
\hline & Hylocharis chrysura ${ }^{(a, b, c)}$ & Kuarahy ava & Picaflor bronceado & PV & 67 \\
\hline \multicolumn{6}{|c|}{ Charadriiformes } \\
\hline Charadriidae & $V_{\text {Vanellus chilensis }}^{(a, b)}$ & Teteu & Tero tero & PV & 33 \\
\hline \multicolumn{6}{|c|}{ Columbiformes } \\
\hline \multirow{5}{*}{ Columbidae } & Columba livia ${ }^{(b)}$ & Pykasu óga & Paloma domestica & PV & 98 \\
\hline & Columbina picui ${ }^{(a, b)}$ & Pyku'i & Tortolita picui & PV & 100 \\
\hline & Columbina talpacoti $^{(a, b)}$ & Pyku'i pytã & Tortolita colorada & PV & 86 \\
\hline & Leptotila verreauxi ${ }^{(c)}$ & Jeruti & Yeruti comun & PV & 64 \\
\hline & Columbina squammata ${ }^{(z)}$ & Pyku'i pini & Palomita escamada & $\mathrm{V}$ & 7 \\
\hline
\end{tabular}




\begin{tabular}{|c|c|c|c|c|c|}
\hline \multicolumn{6}{|l|}{ Coraciiformes } \\
\hline \multirow{2}{*}{ Alcedinidae } & Megaceryle torquata ${ }^{(a, b)}$ & Javatĩ guasu & $\begin{array}{l}\text { Martin pescador } \\
\text { grande }\end{array}$ & PV & 19 \\
\hline & Chloroceryle amazona ${ }^{(z)}$ & Jagua kati & $\begin{array}{l}\text { Martin pescador } \\
\text { mediano }\end{array}$ & $\mathrm{V}$ & 7 \\
\hline \multicolumn{6}{|l|}{ Cuculiformes } \\
\hline \multirow{3}{*}{ Cuculidae } & Crotophaga ani $i^{(a, b)}$ & Ano & Ano chico & PV & 62 \\
\hline & Guira guira $^{(a, b)}$ & Piriringua & Piririta & PV & 55 \\
\hline & Tapera naevia ${ }^{(z)}$ & Chochĩ & Ccrespín & $\mathrm{P}$ & 7 \\
\hline \multicolumn{6}{|l|}{ Falconiformes } \\
\hline \multirow{3}{*}{ Falconidae } & Caracara plancus $^{(b)}$ & Kara kara & Carancho & $\mathrm{V}$ & 10 \\
\hline & Falco sparverius $^{(a)}$ & Kiri kiri'i & Halconcito colorado & $\mathrm{V}$ & $\begin{array}{l}\text { Muestreo } \\
\text { ocasional }\end{array}$ \\
\hline & Milvago chimachima $a^{(a, b)}$ & Kiri kiri & Chimachima & $\mathrm{V}$ & $\begin{array}{l}\text { Muestreo } \\
\text { ocasional }\end{array}$ \\
\hline \multicolumn{6}{|l|}{ Gruiformes } \\
\hline Rallidae & Aramides cajaneus $^{(z)}$ & Chiricoe & Syryco & $\mathrm{P}$ & 5 \\
\hline \multicolumn{6}{|l|}{ Passeriformes } \\
\hline \multirow[b]{2}{*}{ Emberizidae } & Ammodramus humeralis $^{(z)}$ & Manimbe & Cachilo ceja amarilla & PV & 17 \\
\hline & Zonotrichia capensis ${ }^{(a, b, c)}$ & $\begin{array}{l}\text { Che sy } \\
\text { hasy }\end{array}$ & Bendito sea & PV & 100 \\
\hline \multirow[t]{2}{*}{ Fringillidae } & Spinus magellanica ${ }^{(z)}$ & Parachiri & Cabecita negra & PV & 26 \\
\hline & Furnarius rufus ${ }^{(a, b, c)}$ & Ogaraity & Hornero & PV & 100 \\
\hline \multirow[t]{2}{*}{ Furnariidae } & $\begin{array}{l}\text { Lepidocolaptes } \\
\text { angustirostris }^{(a, b)}\end{array}$ & Arapasu ka'atĩ & Chinchero chico & PV & 81 \\
\hline & Phacellodomus ruber ${ }^{(z)}$ & Añumby pytã & Espinero grande & $\mathrm{V}$ & $\begin{array}{l}\text { Muestreo } \\
\text { ocasional }\end{array}$ \\
\hline \multirow[t]{2}{*}{ Hirundinidae } & Progne chalybea ${ }^{(z)}$ & $\begin{array}{l}\text { Mbyju'i } \\
\text { ogarehegua }\end{array}$ & $\begin{array}{l}\text { Golondrina } \\
\text { domestica }\end{array}$ & $\mathrm{P}$ & 7 \\
\hline & Progne tapera $^{(z)}$ & Taperã & Golondrina parda & $\mathrm{P}$ & 7 \\
\hline \multirow{5}{*}{ Icteriade } & Agelaioides badius $^{(z)}$ & Chopi pytã & Tordo músico & PV & 81 \\
\hline & Gnorimopsar chopi $i^{(z)}$ & Chopi & Tordo chopi & PV & 12 \\
\hline & $\begin{array}{l}\text { Icterus } \\
\text { cayanensis }^{(a, c)}\end{array}$ & Guyraumi & Boyerito & PV & 10 \\
\hline & Molothrus bonariensis ${ }^{(a, b, c)}$ & Guyraũ & Tordo renegrido & PV & 100 \\
\hline & Molothrus rufoaxillaris ${ }^{(z)}$ & Arumarã & Tordo pico corto & PV & 10 \\
\hline Mimidae & Mimus saturninus $^{(a, b)}$ & Gyura ñe'ẽngatu & Calandria grande & PV & 60 \\
\hline Passeridae & Passer domesticus ${ }^{(a, b, c)}$ & Guyra tupao & Gorrión & PV & 100 \\
\hline Polioptilidae & Polioptila dumicola ${ }^{(c)}$ & Siritui & Tacuarita azul & PV & 76 \\
\hline Thamnophilidae & Thamnophilus doliatus $^{(a)}$ & Che oro para & Batará rayado & PV & 52 \\
\hline \multirow{5}{*}{ Thraupidae } & $\begin{array}{l}\text { Coryphospingus } \\
\text { cucullatus }^{(a)}\end{array}$ & Guyra pytã'i & Brasita de fuego & PV & 12 \\
\hline & Conirostrum speciosum $^{(z)}$ & Sai & Mielerito azul & PV & 10 \\
\hline & Saltator coerulescens ${ }^{(c)}$ & Havia tyvyta & Pepitero gris & PV & 79 \\
\hline & Volatinia jacarina $^{(b)}$ & Jakarimi & Volatinero & PV & 24 \\
\hline & Euphonia chlorotica $^{(b, c)}$ & Ñandesy & Viví & $\mathrm{P}$ & 7 \\
\hline
\end{tabular}




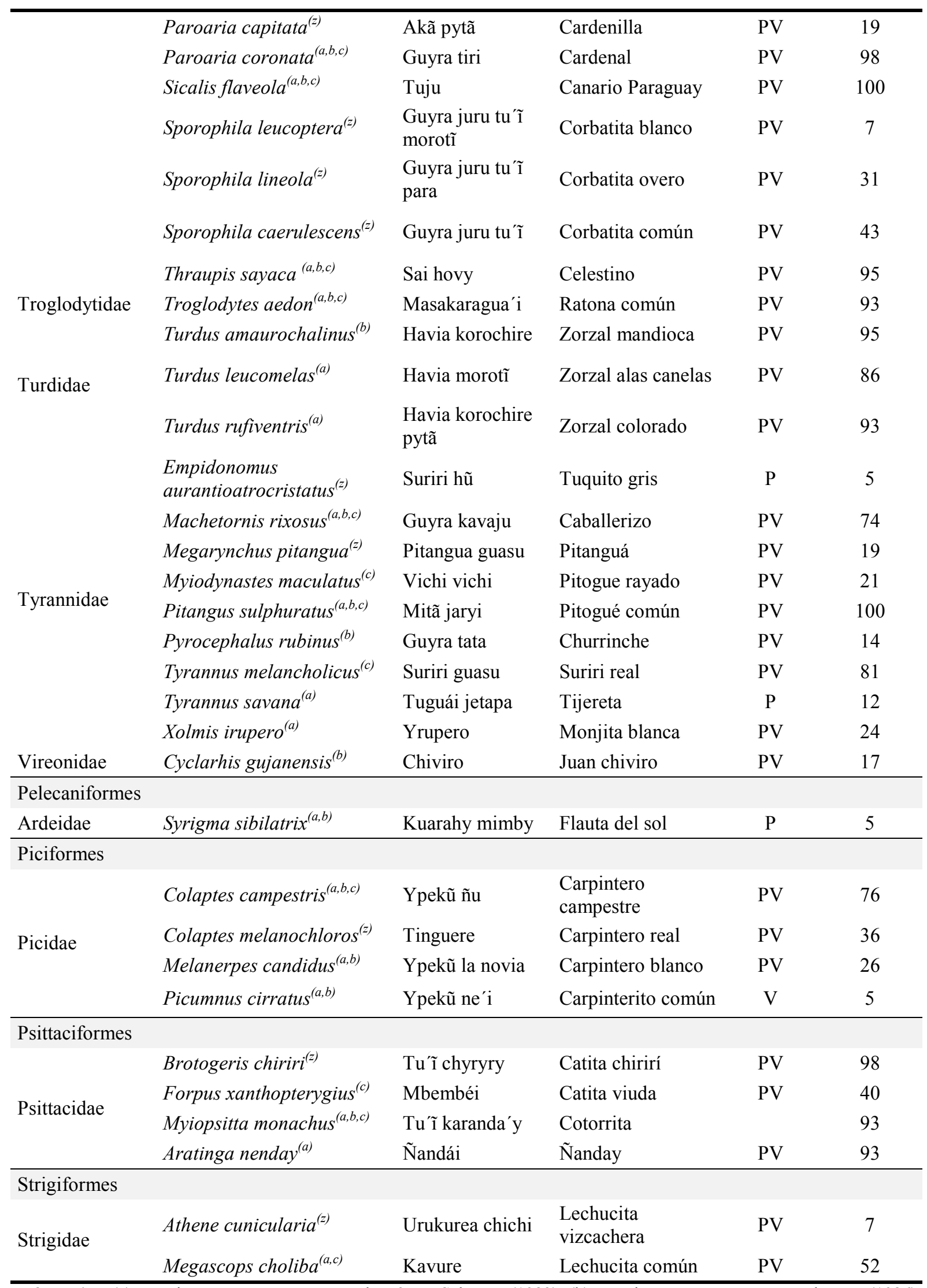

Referencias. (a) = registros en común con Escobar \& De Salomón (1983); (b) = registros en común con Rodríguez (1986); (c) = registros en común con Álvarez et al. (2012); (z) = especies citadas por primera vez; PV = primavera y verano; $\mathrm{P}=$ primavera y $\mathrm{V}=$ verano; $\mathrm{IIR}=$ Índice de importancia relativa. 


\section{Discusión}

La riqueza de aves en el Campus Universitario de la Universidad Nacional de Asunción (77 especies) representa aproximadamente el $11 \%$ de las especies de aves de Paraguay (Guyra Paraguay 2015). La riqueza en el presente trabajo es mayor a las registradas en estudios previos sobre aves del Campus Universitario. Escobar \& De Salomón (1983) registraron 41 especies con un esfuerzo de muestreo de dos años (1981-1983). Rodríguez (1986) registró 42 especies con un esfuerzo de muestreo de un mes (1986) y más recientemente Álvarez et al. (2012) registraron 24 especies con un esfuerzo de muestreo de 21 días (2011).

De las 77 especies de aves, 38 representan registros compartidos con el trabajo de Escobar \& De Salomón (1983), 35 especies son registros compartidos con el trabajo de Rodríguez (1986) y 22 especies se registraron tanto en este estudio como en un trabajo más reciente de Álvarez et al. (2012). Especies citadas en los trabajos previos, no registradas en el presente aporte son: Nothura maculosa, Anumbius annumbi, Thamnophilus caerulescens, Schoenophilax phryganophila, Elenia albiceps, Miarchus tyrannulus, Phacellodomus sibilatrix, Columba picazuro, Veniliornis passerinus y Campylorhynchus turdinus. Sin embargo, se citan a 26 especies por primera vez, lo que representa un aumento en el conocimiento de la riqueza y composición de aves del Campus Universitario (Tabla 1).

En cuanto a la abundancia de especies de aves, los datos actuales se compararon únicamente con el trabajo de Escobar \& De Salomón (1983), debido a que su muestreo fue más exhaustivo. En ese trabajo, la abundancia se basó en la frecuencia de observación. La categoría de abundancia coincide para la mayoría de las especies, excepto Aratinga nenday, Megascops choliba, Megaceryle torquata y Coryphospingus cucullatus, que resultaron en este estudio como escasas o raras, lo que sugiere un declive poblacional. Mientras que las especies Columbina talpacoti, Myiopsitta monachus y Mimus saturninus resultaron en este estudio como abundantes, lo que sugiere en este caso un aumento poblacional de estas especies.

El rol de las áreas verdes urbanas en la conservación de la avifauna ha sido demostrado en varios países de Latinoamérica (Savard et al. 2000). En este trabajo se demuestra, según los registros obtenidos sobre la riqueza, la composición y la abundancia de las especies de aves, que el Campus Universitario es un sitio importante para muchas aves, probablemente porque aún conserva importantes parches de vegetación en relación al área circundante.

\section{Conclusión}

Este trabajo representa una contribución al conocimiento de las aves que habitan el Campus Universitario de la Universidad Nacional de Asunción. Durante el periodo de septiembre de 2014 a marzo de 2015, se registraron 77 especies de aves distribuidas en 27 familias correspondientes a 12 órdenes. En cuanto a la abundancia, un tercio de las especies son consideradas abundantes por su frecuencia de aparición y uso del espacio disponible dentro del Campus Universitario. Las restantes especies probablemente utilizan el campus como parte de su área de distribución, de una forma más esporádica.

\section{Agradecimientos}

Agradecemos a Gloria Delmás y Andrea Weiler por las correcciones y sugerencias sobre el manuscrito. A la Facultad de Ciencias Exactas y Naturales (Universidad Nacional de Asunción) por permitirnos tomar los datos dentro del campus universitario. A los dos revisores anónimos y al editor por la lectura crítica y por las valiosas sugerencias realizadas que permitieron mejorar este manuscrito. 


\section{Bibliografía}

Álvarez, A., Barreto, M., Flores, C. \& Nuñez, S. 2012. Diversidad ornitológica en el predio de la Facultad de Ciencias Exactas y Naturales. San Lorenzo - Paraguay. $30 \mathrm{pp}$.

Avibase, 2015. The world bird database. Consultado el 29/05/2015. Disponible en http://avibase.bsc-eoc.org/avibase.jsp.

Clements, J. F., Schulenberg, T. S., Iliff, M. J., Roberson, D., Fredericks, T. A., Sullivan, B. L. \& Wood, C. L. 2015. The eBird/Clements checklist of birds of the world: v2015. Downloaded from http://www.birds.cornell.edu/clementschecklist/download/

Chace, J. \& Walsh, J. 2006. Urban effects on native avifauna: a review. Landscape and Urban Planning 74: 46-69.

Colwell, R.K. 2013. EstimateS: Statistical estimation of species richness and shared species from samples. Version 9. User's Guide and application published at: http://purl.oclc.org/estimates

De Jesús, A. 2006. Diagnóstico Ambiental en la Facultad de Ciencias Agrarias, UNA Campus San Lorenzo Paraguay.

De la Peña, M. R. \& Rumboll, M. 1998. Birds of Southern South America and Antarctica. Princeton Illustrated Checklist. $304 \mathrm{pp}$.

Escobar, E. \& De Salomón, J. 1983. Contribución al conocimiento de algunas aves que habitan el campus universitario. San Lorenzo - Paraguay.

Faggi, A. \& Perepelizin, P.V. 2006. Riqueza de aves a lo largo de un gradiente de urbanización en la ciudad de Buenos Aires. Revista del Museo Argentino de Ciencias Naturales Bernardino Rivadavia 8(2): 289-297.

Gatto, A., Quintana, F., Yorio, P. \& Lisnizer, N. 2005. Abundancia y diversidad de aves acuáticas en un humedal marino del Golfo San Jorge, Argentina. Hornero 020(02): 141-152.

González-Oreja, J.A., C. Bonache, D. Buzo, A.A. De la Fuente \& L. Hernández. 2007. Caracterización ecológica de la avifauna de los parques urbanos de la ciudad de Puebla (México). Ardeola 54: 53-67.

Guyra Paraguay. 2002. 101 Aves Comunes de Paraguay. Asociación Guyra Paraguay Asunción, Paraguay. 120 pp.

Guyra Paraguay. 2015. Síntesis del estado de conservación de las aves en Paraguay. Consultado el 20/05/2015. Disponible en http://www.guyra.org.py/index.php?lang=es.

IUCN Red List of Threatened Species. Version 2015.2 Consultado el 10 de mayo de 2015. Disponible en www.iucnredlist.org.

Juri, M. \& Chani, J. 2009. Variación estacional en la composición de las comunidades de aves en un gradiente urbano. Ecología Austral 19: 175-184.

Leveau, L. M. \& Leveau, C. M. 2004. Comunidades de aves en un gradiente urbano de la ciudad de Mar del Plata, Argentina. Hornero 19(1): 13-21.

Londoño-Betancourth, J. C. 2011. Una mirada a la diversidad ornitológica de Pereira. Boletín Científico Museo de Historia Natural 15(1): 84-103.

Maragliano, R.E., Marti, L.J., Ibáñez, L.M. \& Montalt,i D. 2009. Comunidades de aves urbanas de Lavallol, Buenos Aires, Argentina. Acta Zoológica Lilloana 53(1-2): 108-114.

Marín, O. 2005. Avifauna del Campus de la Universidad del Quindío. Lima - Perú Boletín Sao. 15(2): 42 - 59.

Moreno, C.E. 2001. Métodos para medir la biodiversidad. M\&T-Manuales y Tesis moSEA, vol. 1.

Narosky, T. \& Yzurieta, D. 2006. Guía para la Identificación de las Aves de Paraguay $1^{\mathrm{a}}$ ed. - Buenos Aires. 240 pp. 
Ralph, C., Geupel, G., Pyle P., Martin, T., De Sante, D. \& Milá, B. 1996. Manual de métodos de campo para el monitoreo de aves terrestres. General Technical Report PSW-GTR-159, USDA Forest Service, Albany. 46pp.

Ramirez, A. 2005. Estudio de la Facultad de Arquitectura en su relación con el campus (U.N.A). San Lorenzo - Paraguay. 0904. ARQ.01.

Rodríguez, E.J. 1986. Estudio estadístico de la población de aves el campus universitario. 45pp. (ICB) Instituto de Ciencias Básicas. San Lorenzo Paraguay Informe técnico no publicado.

Savard, J., Clergeau, P. \& Mennechez, G. 2000. Biodiversity concepts and urban ecosystems. Landscape and UrbanPlanning 48:131-142.

Stiles, F.G. 1990. La avifauna de la universidad de Costa Rica y sus alrededores a través de veinte años (1968-1989). Revista de Biología Tropical 38(2B): 361-381.

Takano, F. \& Castro, N. 2007. Avifauna en el campus de la Universidad Nacional Agraria la Molina (UNALM), Lima Perú. Consultado el 15/04/2015. Disponible en http://www.redalyc.org/articulo.oa?id=34160217.

Recibido: noviembre 2015

Aceptado: mayo 2016 